rations is unlikely to be any less problematical than under Bill Clinton which witnessed the failures, disasters and horrors of Somalia, Srebrenica and Rwanda. And she is likely to emphasise nuclear nonproliferation and downplay disarmament.

John McCain, finally, is the only one of the three to know firsthand what war means. The deliberate misrepresentation of his 100-years in Iraq comment notwithstanding, therefore, he could be more cautious than Clinton but because of his party less restrained than Obama in defending US interests with force. He has based his campaign on winning the war in Iraq and not losing focus from the war on terrorism. He seems more interested in cooperating with a coalition or league of democracies than giving priority to the United Nations. ${ }^{31}$ Based on that, like Bush he would most likely differentiate between USfriendly and US-hostile regimes possessing or pursuing nuclear weapons, enlisting the former as strategic partners and allies

31 See John McCain, “An Enduring Peace Built on Freedom," Foreign Affairs 86:6 (November/December 2007), pp. 19-34. while sanctioning the latter as threats to world peace. But he could also offer Reaganesque surprises if the idea of a nuclear-weapon-free world propounded by four former republican heavyweights grabs his attention. But neither by personal instinct nor by party leanings is McCain likely to pursue an activist agenda with respect to UN peace operations and climate change. Moreover, as he is the least likely of the three to serve for a second term (based simply on age), he may have the least longer term impact.

Regardless of who becomes president, to regain its former status as a good international citizen, the US should reinvest diplomatic assets in the United Nations, reassert its former role as the champion-in-chief of the global human rights norm, "re-sign" and ratify the Rome Statute of the ICC that was "unsigned" by Bush in 2002, reaffirm firm adherence to the Geneva and UN torture conventions, ratify the CTBT, and assume the leadership role in negotiating a post-Kyoto climate deal.

\title{
Die Strategien der Administrationen Bill Clinton und George W. Bush gegenüber dem Iran im Vergleich
}

\author{
Bernd W. Kubbig und Sven-Eric Fikenscher*
}

\begin{abstract}
This article examines the policies of the Clinton and George W. Bush administrations towards the Islamic Republic of Iran. It analyses continuity and change and terms of both the "Grand Strategy" and operational US policy with its objectives and preferred instruments. Since 2005, a significant feature of the second term of the Bush administration has been the competing policies of the neoconservatives and the conservative realists. This contribution concludes that the latter group has prevailed so far mainly due to the catastrophic situation in Iran's neighbour Iraq. From today's perspective it seems that during the remaining months of the current US government, neither a "regime change" nor a military option in the form of a bombardment of Iranian nuclear facilities is realistic (both objectives have been favoured by "Neocons"). Nevertheless, the conservative realists led by Condoleezza Rice, who have increasingly supported a policy of containment, reject the option of selective and unconditional engagement with Tehran via direct diplomacy - an approach which had been favoured by the outgoing Clinton administration.
\end{abstract}

Keywords: Amerikanische Außenpolitik, Islamische Republik Iran, Sanktionen, Nichtverbreitung, Eindämmungsstrategie, „regime change“, Neokonservative, Clinton-Administration, George W. Bush-Administration

\section{Kontinuität und Neuerungen in der amerika- nischen Iran-Politik}

$\mathrm{P}$ olitische Stabilität, gesicherter Zugang zum Öl sowie der verbriefte Schutz Israels und der arabischen Verbündeten - diese Trias umfasst die grundlegenden Interessen und Ziele, die die Vereinigten Staaten bereits während des Ost-

\footnotetext{
PD Dr. Bernd W. Kubbig ist Projektleiter an der Hessischen Stiftung Friedensund Konfliktforschung (HSFK) und Privatdozent an der Johann Wolfgang Goethe-Universität in Frankfurt/M. Sven-Eric Fikenscher ist studentischer Mitarbeiter in der HSFK-Projektgruppe Raketenabwehrforschung International. - Dieser Beitrag beruht partiell auf der demnächst erscheinenden Studie: Bernd W. Kubbig (in Zusammenarbeit mit Sven-Eric Fikenscher), Die Iran-Politik der Regierung Bush ab 2005: Brüche - rivalisierende Konzepte - Durchsetzungschancen (HSFK-Report), Frankfurt/M. 2008. Der Aufsatz wurde referiert (peer-review).
}

West-Konflikts kontinuierlich in der Konfliktregion Naher und Mittler Osten/Golf ${ }^{1}$ verfolgt haben. Diese Interessen und Ziele stehen für Washington unter der übergeordneten Leitlinie, mit allen Mitteln zu verhindern, dass der Golf unter die Kontrolle rivalisierender Mächte fällt. ${ }^{2}$ Die USA setzten dabei jahrzehntelang auf realpolitische Elemente, also primär auf eine Eindämmungspolitik gegenüber ihren Gegnern. Im Rahmen dieser

1 Wir meinen in der Regel die gesamte Region, wenn wir im Folgenden aus sprachlichen Gründen oft vom Nahen bzw. vom Mittleren Osten sprechen.

2 Siehe beispielhaft: A National Security Strategy of Engagement and Enlargement, Washington, D.C. 1995, 30. - Grundlegend für Viele: Steven L. Spiegel, The Other Arab-Israeli Conflict. Making America's Middle East Policy, from Truman to Reagan, Chicago, IL/London 1985; William B. Quandt, Peace Process. American Foreign Policy and the Arab-Israeli Conflict Since 1967, Washington D.C. u.a. 1993; David W. Lesch (Hrsg.), The Middle East and the United States. A Historical and Political Reassessment, Boulder, CO/Oxford 1996. 
„containment“-Strategie nahm der Iran unterschiedliche Rollen ein. Das diktatorische Schah-Regime fungierte rund ein Vierteljahrhundert lang als Bündnismacht. Es sollte, von Washington beständig mit den modernsten Waffen ausgerüstet, vor allem die expandierende Sowjetunion in jenem ölreichen Krisengebiet in Schach halten. Mit der Machtübernahme des Fundamentalisten Ayatollah Chomeini wurde aus dem Verbündeten ein kategorischer Gegner, ja, ein feindliches Regime, das die US-Interessen auf vielfältige Weise durchkreuzte. In der Ära Clinton, in den 1990er Jahren, bestand die Variante der Eindämmungspolitik darin, die beiden regionalen Hauptrivalen - neben der Islamischen Republik war dies der Irak Saddam Husseins - gleichzeitig in ihrem außenpolitischen Einfluss zu begrenzen („dual containment“). Diese Strategie beruhte auf den außenpolitischen Instrumenten klassischer regionaler Bündnispolitik und unilateraler Sanktionen. Hinzu kam eine Bereitschaft zum Dialog, die sich gegen Ende der Amtszeit Clintons in einem konkreten Angebot zur Normalisierung des bilateralen Verhältnisses äußerte.

Mit dem Amtsantritt von George W. Bush im Januar 2001 waren neue Akzente in der amerikanischen Iran-Politik verbunden. Die Administration stigmatisierte die Islamische Republik in einem unter Clinton nicht gekannten Ausmaß ${ }^{3}$ und titulierte sie als Teil der „Achse des Bösen“. Sogar ein Regimewechsel („regime change“) in Teheran rückte als Ziel der US-Politik in den Bereich des Möglichen; die Bush-Doktrin führt präventive Militärschläge ${ }^{4}$ als neues Instrument im Kampf gegen „Schurkenstaaten“ mit Programmen zur Herstellung von Massenvernichtungswaffen auf. Die 2006 veröffentlichte zweite „National Security Strategy“ (NSS) der Bush-Administration geht noch weiter und erklärt „die Freiheit der iranischen Bevölkerung“ zur Grundvoraussetzung für die Lösung des bilateralen Konflikts. ${ }^{5}$

Vor diesem Hintergrund entwickelt dieser Aufsatz die folgende Forschungsfrage: Wie gestaltete sich die Iran-Politik von der Clinton- zur Bush-Administration sowie in der Ära Bush im Hinblick auf a) die „Grand Strategy“ (Gesamtstrategie, -konzept), wie sie vor allem in den „Nationalen Sicherheitsstrategien“ ihren Ausdruck findet; b) die tatsächlich verfolgten Zielsetzungen und c) die bevorzugten Instrumente, mit denen diese Ziele erreicht werden sollen.

Im Hinblick auf die Regierung George W. Bush bedeutet dies, die beiden außenpolitischen Hauptausrichtungen (konservative Realisten und Neokonservative) zu berücksichtigen. Während sich beide Gruppierungen bei der Iran-Politik in der ersten Amtszeit neutralisierten, brachte die US-Administration nach 2005 eine Reihe von Maßnahmen gegenüber der Islamischen Republik auf den Weg, die das unterschiedliche Profil beider Konzepte offenbaren. Wie wir im Einzelnen darstellen werden, bezieht sich dies sowohl auf die „Grand Strategy" als auch auf die Zielsetzungen und Mittel.

3 Auch die Clinton-Administration bezeichnete den Iran des Öfteren als „Schurkenstaat", strich diesen Begriff aber im Juni 2000 aus ihrem außenpolitischen Vokabular (Frankfurter Allgemeine Zeitung, 21.6.2000).

4 Die NSS von 2002 selbst spricht von der Option, „prä-emptiv“ zu handeln (The National Security Strategy of the United States of America, Washington, D.C. 2002, 6)

5 The National Security Strategy of the United States of America, Washington, D.C. $2006,20$.

\section{Die „dual containment“-Politik Clintons und die Annäherungsversuche gegenüber Teheran}

Nach dem Zusammenbruch der Sowjetunion richtete sich Washingtons Hauptinteresse einerseits auf Osteuropa, wo es die neu entstandenen Demokratien zu stabilisieren galt, und andererseits auf die Russische Föderation, die sich zu einer möglichst ausgeprägten Demokratie westlichen Zuschnitts entwickeln sollte. Ein weiteres Ziel war es, die gesamte Region in die globalen Handels- und Finanzinstitutionen einzubinden. Die Verbreitung von Demokratie und Marktwirtschaft kennzeichnete die „Grand Strategy“ unter Clinton. Dieser maßgebliche außenpolitische Grundsatz, der erstmals in der 1995 vorgelegten „Nationalen Sicherheitsstrategie“ seinen Ausdruck fand, ${ }^{6}$ galt jedoch nicht für den Nahen Osten. Realpolitische Erwägungen bestimmten weiterhin die amerikanische Ordnungspolitik gegenüber dieser Region. Im Vordergrund stand zum einen das Bemühen, zwischen Israel und seinen arabischen Nachbarn einen dauerhaften Frieden zu vermitteln; zum anderen galt es auch jetzt, das eingangs erwähnte übergeordnete Ziel umzusetzen, nämlich den Aufstieg rivalisierender Mächte in der Region zu unterbinden. Gegenüber Teheran und Bagdad lief dies auf eine Politik der doppelten Eindämmung („dual containment“) hinaus. ${ }^{7}$ Im Hinblick auf den Iran wollte Washington darauf hinwirken, dass Teheran sein Verhalten in vier Bereichen grundlegend ändert: das Streben nach Massenvernichtungswaffen und Raketen; die Unterstützung von Terroristen und Gruppierungen, die den Nahost-Friedensprozess ablehnen; die Unterminierung anderer Regierungen in der Region; und die Verletzung von Menschenrechten im eigenen Land. ${ }^{8}$

Die Strategie zur Eindämmung Teherans bestand aus zwei Elementen: dem Auf- und Ausbau von kooperativen Sicherheitsbündnissen in der Region (einschließlich neuer Waffenlieferungen $^{9}$ ) sowie aus einer strikteren Sanktionspolitik. Der Regierung Clinton gelang es, einen Teil der bestehenden Abkommen über eine begrenzte Stationierung amerikanischer Truppen mit den alliierten Golfstaaten zu erneuern bzw. auszubauen. ${ }^{10}$ Ohne dass dies in der „Nationalen Sicherheitsstrategie" vorgesehen war, entwickelten sich die Sanktionen zu einem zweiten Element der „containment“-Politik. Die Regierung Clinton verschärfte die bisherigen Strafmaßnahmen und Importbeschränkungen beträchtlich, als Teheran seinen Öl- und Gassektor für ausländische Investitionen öffnete. Washington reagierte 1995 mit einem Investitionsverbot für amerikanische Firmen im Energiebereich sowie mit einem umfassenden Han-

6 Michael Cox, Wilsonianism Resurgent? The Clinton Administration and the Promotion of Democracy, in: Ders./G. John Ikenberry/Takashi Inogushi (Hrsg.): American Democracy Promotion. Impulses, Strategies and Impacts, Oxford/New York, NY 2000, 218-239, 232.

7 A National Security Strategy of Engagement and Enlargement, Washington, D.C. 1995, 30; siehe hierzu: Raymond Tanter, Rogue Regimes. Terrorism and Proliferation, Houndmills u.a. 1998, 77-80; Robert S. Litwak, Iraq and Iran. From Dual to Differentiated Containment, in: Robert J. Lieber (Hrsg.): Eagle Rules? Foreign Policy and American Primacy in the Twenty-First Century, Upper Saddle River, NJ 2002, 173-193. - Kritisch zum Konzept der doppelten Eindämmung: F. Gregory Gause III, The Illogic of Dual Containment, in: Foreign Affairs, Jg.73, Nr.2, 1994, 56-66.

8 A National Security Strategy of Engagement and Enlargement, Washington, D.C. $1995,31$.

9 Ebd.

10 Martin S. Indyk, US Policy Priorities in the Gulf: Challenges and Choices, in: The Emirates Center for Strategic Studies and Research (Hrsg.): International Interests in the Gulf Region, Abu Dhabi 2004, 103-130, 108f. 
delsboykott. ${ }^{11}$ Der Iran-Libya Sanctions Act (ILSA) von 1996, auf Betreiben des Kongresses zustande gekommen, ging noch einen Schritt weiter. Dieses Gesetz sieht sogar Sekundärsanktionen gegen ausländische Firmen oder Personen vor, die mehr als 20 Mio. US-Dollar pro Jahr in den Öl- und Gassektor der Islamischen Republik investieren. ${ }^{12}$

Die Regierung Clinton erklärte zwar stets ihre Bereitschaft zu einem Dialog mit Teheran, ${ }^{13}$ aber erst nach der Wahl des moderaten Mohammed Chatami zum Staatspräsidenten, 1997, ging die US-Administration auf die neue Führung in Teheran zu. Chatami hatte seinerseits bereits vorher im Rahmen des von ihm proklamierten „Dialogs der Zivilisationen“ ein vorsichtiges Interesse an der Kontaktaufnahme mit den Vereinigten Staaten - zunächst durch Austauschprojekte - signalisiert. Die Clinton-Administration reagierte mit einem zeitlich gestaffelten Programm, das überwiegend symbolische Maßnahmen beinhaltete. Ihr größtes Entgegenkommen bestand darin, dass sie in ihrem letzten Amtsjahr die Einfuhrbeschränkungen für Dörrobst, Pistazien, Kaviar und Teppiche aufhob. ${ }^{14}$ Die scheidende Regierung Clinton sandte ferner der iranischen Führung einen umfassenden Vorschlag zur Normalisierung der amerikanisch-iranischen Beziehungen zu. ${ }^{15}$ Washington hatte seine realpolitische Linie gegenüber Teheran erstmals um den Aspekt der angestrebten Direktdiplomatie ergänzt. Führende Politiker suchten zudem den persönlichen Kontakt zur iranischen Regierung. Die damalige Außenministerin Madeleine Albright resümierte rückblickend auf ihr letztes Amtsjahr 2000:

„In meiner Rede [...] lud ich es [das Land Iran, die Autoren] abermals ein, in einen offiziellen Dialog einzutreten. [...] Durch unser bedingungsloses Dialogangebot schoben wir dem Iran den schwarzen Peter zu, der jetzt erklären musste, warum er nicht einmal bereit war, über unsere Differenzen zu sprechen. Damit legten wir den Grundstein für offizielle Gespräche, falls und wann immer sie möglich werden sollten." 16

Die von Albright erwähnte Bedingungslosigkeit änderte allerdings nichts an dem von der Clinton-Administration angestrebten Verhandlungsergebnis. Denn die US-Regierung bestand auf ihrer Forderung, dass die Islamische Republik ihr Verhalten in den vier genannten Punkten ändert. Zu diesen Konditionen war Teheran jedoch in der Ära Clinton nicht zu Gesprächen bereit. ${ }^{17}$

11 Patrick Clawson/Michael Rubin, Eternal Iran. Continuity and Chaos, New York, NY u.a. 2005, 124.

12 Kinka Gerke, Die unilaterale Versuchung: Die Sanktionen der USA gegen die Handelspartner Kubas, Irans und Libyens und ihre Auswirkungen auf das Welthandelsregime (HSFK-Report 2/1997), Frankfurt/M. 1997, $29 \mathrm{f}$.

13 In der „National Security Strategy” von 1995 heißt es dazu: „We remain willing to enter into an authoritative dialogue with Iran to discuss the differences between us." A National Security Strategy of Engagement and Enlargement, Washington, D.C. 1995, 31.

14 Kenneth M. Pollack, The Persian Puzzle. The Conflict Between Iran and America, New York, NY 2005, 319ff; Madeleine K. Albright, Madame Secretary. Die Autobiographie, München 2003, 393.

15 Ali M. Ansari, Confronting Iran. The Failure of American Foreign Policy and the Roots of Mistrust, London 2006, 178.

16 Albright 2003: 393f.

17 Pollack 2005: 341

\section{Die Iran-Politik der Bush-Administration im Kontext ihrer "Grand Strategy"}

\subsection{Das Gesamtkonzept und die rivalisierenden Zielsetzungen der Konservativen}

In der außenpolitischen „Grand Strategy“ George W. Bushs finden sich die Grundlinien der Iran-Politik indirekt in der „Nationalen Sicherheitsstrategie“ von 2002 bzw. direkt in der aktualisierten Version von 2006. Die NSS von 2002 kodifizierte mit der Bush-Doktrin vorbeugender militärischer Schläge gegen "Schurkenstaaten“ („rogue states“) erstmals ein Kernelement des neokonservativen Sicherheitsdenkens. ${ }^{18}$ Die Definition von „rogue states“ lässt sich auf die Islamische Republik anwenden, die aber nicht explizit genannt wurde. Die ebenfalls von den „Neocons“ dominierte Fassung von 2006 legte sogar einen Wechsel der Führung in Teheran als Ziel amerikanischer Politik nahe. ${ }^{19}$ Sie verknüpfte die vielfältige iranische Bedrohung mit dem Regime selbst. Die Iran-bezogene Bedrohungsanalyse veränderte sich somit von Clinton zu Bush in einem wesentlichen Punkt: Für die „Nationale Sicherheitsstrategie“ von 1995 ging - ganz in der realpolitischen Tradition - die Gefahr nahezu ausschließlich vom aggressiven Außenverhalten Teherans und dessen militärischen Fähigkeiten aus. Entsprechend war es das Ziel, auf dieses Verhalten einzuwirken ${ }^{20}$ und nicht das Regime in Teheran selbst zu verändern.

Das Ziel neokonservativer Iran-Politik war in der zweiten Amtszeit Bush zum einen eingebunden in ihre umfassende weltordnungspolitische Vision, „die Tyrannei“ weltweit zu beenden. ${ }^{21}$ Zum anderen war es mit dem Grundanliegen verknüpft (das auch die konservativen Realisten teilten), die Monopolposition der USA gegenüber Herausforderern mit hegemonialen Bestrebungen zu erhalten. Dem traditionellen weltordnungspolitischen Hauptziel der konservativen Realisten - Stabilität - erteilte die NSS von 2006 eine moralisch-demokratiepolitisch begründete Absage, indem sie als „Illusion“ gebrandmarkt wurde; unter ihrem Deckmantel konnten Repression und Extremismus als „Krankheiten“ gedeihen. ${ }^{22}$ Die internationale Isolierung des Irans - das von den Realpolitikern in der BushAdministration proklamierte Ziel - fehlte völlig in der „Nationalen Sicherheitsstrategie“ vom März 2006.

Mit den Neokonservativen und konservativen Realisten sind zwei in der Bush-Administration miteinander rivalisierende Akteursgruppen genannt, deren Profil hier nur in Stichworten konturiert werden kann. Für die Neokonservativen sind Konflikte - wie oben am Beispiel des Irans erwähnt - grundsätzlich regimeabhängig. Die konsequente Antwort ist die notfalls gewaltsame globale Verbreitung von Demokratie. Im Sinne dieser außenpolitischen Maxime setzten sich Ex-Verteidigungsminister Donald Rumsfeld und sein damaliger Under Secretary of Defense

18 Siehe hierzu vor allem: The National Security Strategy of the United States of America, Washington, D.C. 2002, 6.

19 The National Security Strategy of the United States of America, Washington, D.C. $2006,20$.

20 A National Security Strategy of Engagement and Enlargement,, Washington, D.C. 1995, 31

21 The National Security Strategy of the United States of America, Washington, D.C. $2006,3$.

22 Ebd., 38. 
for Policy Douglas Feith in der ersten Amtszeit Bushs dafür ein, dass ein Regimewechsel in der Islamischen Republik zur offiziellen Linie der US-Administration wurde. ${ }^{23}$ Dies kann einen gewaltsamen Sturz der iranischen Führung sowie die Stärkung oppositioneller Kräfte einschließen. Nachdem zahlreiche „Neocons“ die Administration verlassen haben, repräsentiert Vize-Präsident Richard Cheney das Gravitationszentrum dieser Grundausrichtung.

Für die konservativen Realisten ist Stabilität das zentrale weltordnungspolitische Anliegen. Ein „regime change“ sollte nicht das Ziel der amerikanischen Iran-Politik sein, sondern ein verändertes (Außen-)Verhalten der Führung in Teheran ${ }^{24}$ - das war die Gegenposition der konservativen Realisten an der Spitze des State Department um Colin Powell und seinem Stellvertreter Richard Armitage zu den Akteuren im Pentagon. Sie befürworteten eine traditionelle Eindämmungspolitik gegenüber der Islamischen Republik, wie sie die Regierung Clinton praktizierte. Zum klassischen Kanon der von ihnen bevorzugten außenpolitischen Instrumente, die entsprechend des „nationalen Interesses“ der Vereinigten Staaten zum Einsatz kommen sollen, gehören (verschärfte) unilaterale und internationale Sanktionen, der Aufbau einer Allianz von Gegenmächten („,counterbalancing") sowie ggf. eine selektive Einbindung und direkte Gespräche. In der zweiten Amtszeit Bushs sind Außenministerin Condoleezza Rice und Verteidigungsminister Robert Gates die Exponenten des konservativ-realistischen Flügels.

\subsection{Militärische Optionen und Demokratieförde- rung: Die Neokonservativen in der Defensive}

Nach den Kriegen in Afghanistan und im Irak war die iranische Elite dankbar, dass die Erzfeinde in Afghanistan und im Irak besiegt waren. Gleichzeitig fürchtete sie jedoch, da ihr Land durch die Präsenz von US-Truppen in den benachbarten Staaten umzingelt war, dass sie „als nächste an der Reihe“ sein würde. ${ }^{25}$ Im Falle einer positiven Entwicklung im Irak hätten zumindest die „Neocons“ damals für den Einsatz militärischer Gewalt plädieren können. Dies hätte eine Invasion durch Bodentruppen, aber auch die gezielte Bombardierung von Nuklearanlagen bedeuten können. Nach 2005 verstand man aufgrund der desaströsen Lage im Zweistromland, durch die die amerikanischen Streitkräfte gebunden waren, unter militärischen Optionen im Regelfall gezielte Angriffe auf atomare Einrichtungen in der Islamischen Republik. Laut David Wurmser, einem engen Berater des Vize-Präsidenten in Sicherheitsfragen, drängte Cheney im Sommer 2007 auf eine baldige Entscheidung über einen Militärschlag gegen den Iran. ${ }^{26}$

23 Leon Hadar, Target: Tehran? By placing Iran on the Axis of Evil, Bush made an enemy of a would-be ally, in: The American Conservative, 22.11.2004.

24 So Armitage, in: 108/1 U.S. Congress, Senate, Committee on Foreign Relations: Iran: Security Threats and U.S. Policy, Hearing, 28.10.2003, Washington, D.C. 2004, 13.

25 So der US-Sondergesandte für Afghanistan, James Dobbins, in: 110/1 U.S. Congress, House of Representatives, Committee on Oversight and Government Reform, Subcommittee on National Security and Foreign Affairs: Iran: Reality, Options and Consequences, Part 2: Negotiating with Iranians: Missed Opportunities and Paths Forward, Hearing, 7.11.2007 (unautorisiertes Transkript), 4.

26 International Herald Tribune, 16./17.6.2007.
Damit stieß er auf den Widerstand der konservativen Realisten. In ihrem Iran-Konzept sind militärische Optionen anders verortet. Sie fungierten als Druckmittel, um die Bush-Administration dabei zu unterstützen, dass im UN-Sicherheitsrat möglichst harte Sanktionen gegenüber Teheran verabschiedet wurden. Bis Ende 2007 war unklar, welches der beiden konservativen Konzepte sich durchsetzen würde. Ab Herbst wuchs der Widerstand innerhalb der Bush-Administration gegen Bombardements. Mit Admiral William Fallon, dem damaligen Befehlshaber des „Central Command“, warnte ein führender Repräsentant der US-Streitkräfte Ende September 2007 vor den Risiken von Militärschlägen. ${ }^{27}$ Im November stellte Fallon klar, dass die amerikanischen Streitkräfte als Antwort auf die kriegerische Rhetorik Teherans keinen Militärangriff vorbereiteten. ${ }^{28}$ Am 3. Dezember legten alle 16 US-Geheimdienste eine neue Iran-bezogene „National Intelligence Estimate“ vor. Die zentrale Aussage dieser offiziellen Gefahreneinschätzung lautete, dass das Regime sein militärisches Atomprogramm bereits im Jahr 2003 eingestellt habe. ${ }^{29}$ Diese Bewertung, die Teheran teilweise entlastete, löste im politischen Washington ein Beben aus. Denn sie relativierte die vorherrschende Einschätzung, vom Iran gehe eine akute Bedrohung aus. ${ }^{30}$ Die Rahmenbedingungen für Militärschläge haben sich insgesamt verschlechtert. Die Mehrheit der US-Öffentlichkeit will den (Atom-)Konflikt mit dem Iran ohne Gewalt lösen. ${ }^{31}$ Zudem artikulierte sich in beiden Häusern des Kongresses zunehmend Widerstand gegen die Bombardierung iranischer Einrichtungen. An die Stelle eines „regime change“ ist, ganz im Sinne realpolitischer Konzepte, die Isolierung des Irans mit der Präferenz für Sanktionen getreten. ${ }^{32}$ Kurzfristig geplanten Militäroperationen der USA dürfte damit der Boden entzogen sein. Sie sind in der restlichen Amtszeit Bushs wohl keine reale Option mehr.

Divergenzen zwischen dem neokonservativen und dem konservativ-realistischen Flügel gab es auch im Hinblick auf die Maßnahmen zur Demokratieförderung. Aus neokonservativer Sicht ließ sich ein Regimewechsel auch durch gezielte, kurzfristig durchzuführende und ggf. gewaltsame US-Maßnahmen zur Unterstützung oppositioneller Kräfte erreichen. ${ }^{33}$ Eine umfassendere Initiative zur Demokratisierung des Irans präsentierte Anfang 2006 mit Condoleezza Rice jedoch eine führende konservative Realistin. Diese Initiative beinhaltete in erster Linie erhöhte Finanzmittel und war jetzt in eine Langzeitstrategie eingebettet. Demnach sollten die Iraner die Veränderungen in ihrem Land selbst in die Hand nehmen; externen Akteuren wie den USA kam hierbei damit eine lediglich unterstützende Funktion zu. Diese Agenda grenzte sich somit klar von der neokonservativen Position ab. Die einzelnen Schritte zum fried-

27 Financial Times, 24.9.2007.

28 Financial Times, 12.11.2007.

29 National Intelligence Estimate, Iran: Nuclear Intentions and Capabilities, Washington, D.C. 2007, 9

30 Ausführlicher hierzu: Bernd W. Kubbig, Raketenabwehr in Europa - erhöhte Sicherheit oder neue Rüstungswettläufe? Problemanalyse und Problemlösungen, Raketenabwehrforschung International, Bulletin Nr.61, 2007/08, www.hsfk.de/fileadmin/con-tent-ABM/BU_61_BWK.pdf (letzter Zugriff: 27.3.2008).

31 Siehe hierzu die Übersicht zu den einzelnen Umfragen unter www.pollingreport.com/iran.htm.

32 Siehe in diesem Zusammenhang etwa die vorgelegten Versionen des Iran Nonproliferation Amendments Act of 2005; des Iran Freedom and Support Act; des Iran Sanctions Extension Act of 2006 und der Gesetzesvorlage To prohibit the use of funds for military operations in Iran.

33 The New York Times, 15.2.2006. 
lichen Regimewandel hoben ferner darauf ab, die Führung in Teheran zu schwächen. ${ }^{34}$ Rice selbst begründete die Initiativen mit einem Verweis auf den realpolitischen Interessen-Grundsatz: „We find it in our interest now [...] to see if we can't engage the Iranian population. “35

Nach Rice' Präsentation bewilligte der Kongress 66,1 Mio. USDollar an zusätzlichen Mitteln für das Haushaltsjahr 2006. Der Löwenanteil war für die Förderung oppositioneller Radio- und Fernsehprogramme bestimmt. Von den 25 Mio. US-Dollar, die für die Förderung oppositioneller Kräfte im Iran eingeplant waren, konnte man von amerikanischer Seite bis Ende Mai 2007 nur einen Bruchteil - nämlich weniger als zwei Millionen - an Einrichtungen im Iran überweisen. ${ }^{36}$ Die Angst vor den offenbar zunehmenden Repressionen des Regimes in Teheran, die zumindest teilweise auch eine Reaktion auf die amerikanische Demokratisierungsinitiative waren, ${ }^{37}$ dürfte zum Fehlschlag des Programms beigetragen haben. Denn die Führung in Teheran hält auch dieses Demokratisierungskonzept für eine verdeckte „regime change“-Kampagne, solange die US-Regierung dieses Ziel nicht von ihrer außenpolitischen Agenda nimmt. Dennoch setzt die Bush-Administration ihre Politik unbeirrt fort. Im Budgetjahr 2008 beantragte sie fast 109 Mio. US-Dollar für Programme zur Demokratieförderung im Iran, von denen 50 Mio. US-Dollar direkt in den Golfstaat fließen sollten. ${ }^{38}$

\subsection{Sanktionen und Allianzpolitik als Mittel zur Eindämmung des Irans: Die konservativen Realisten im Aufwind}

Die Bush-Administration hatte es im Kontext des AfghanistanKrieges mit einer Führung in Teheran zu tun, die mit Washington selektiv kooperierte. ${ }^{39}$ Im Frühjahr 2003 machte Teheran einen noch weitreichenden Vorschlag, den sie der Bush-Administration über die Schweizer Botschaft übermittelte. Er sah vor, alle wichtigen und strittigen Themen in eigens eingerichteten Arbeitsgruppen zur Sprache zu bringen, um auf diesem Wege das bilaterale Verhältnis zu normalisieren. Die Bush-Regierung lehnte diese Offerte, die an den Vorschlag der scheidenden Clinton-Administration zur Normalisierung der US-iranischen Beziehungen erinnert, insbesondere auf Druck der "Neocons“ ab. ${ }^{40}$ Auch die Bemühungen der Europäer, die ab 2003 bestrebt waren, Teheran durch angebotsorientierte Verhandlungen zu einem Verzicht auf die Urananreicherung zu bewegen, ver-

34 Hadar 2004.

35 The Boston Globe, www.boston.com/news/nation/washington/ articles/2006/02/16/Rice_wants_funds_for_democracy_initiative_in_Iran/ ?page=2 (letzter Zugriff: 28.8.2007).

36 National Iranian American Council (NIAC), US-Iran News, www. niacouncil.org/index.php?option $=$ com_content\&task $=$ view $\& i d=819 \&$ Itemid=2 (letzter Zugriff: 13.8.2007).

37 Michael McFaul/Abbas Milani/Larry Diamond: A Win-Win U.S. Strategy for Dealing with Iran, in: The Washington Quarterly, Jg.30, Nr.1, 2006/07, 121$138,133$.

38 Freedom House, Supporting Freedom's Advocates? An Analysis of the Bush Administration FY 2008 Budget Request for Democracy and Human Rights, Washington, D.C. 2007, 27.

39 So James Dobbins, in: 110/1 U.S. Congress, House of Representatives, Committee on Oversight and Government Reform, Subcommittee on National Security and Foreign Affairs: Iran: Reality, Options and Consequences, Part 2: Negotiating with Iranians: Missed Opportunities and Paths Forward, Hearing, 7.11.2007 (unautorisiertes Transkript), 4.

40 So Hillary Mann Leverett, ehemals Director for Iran and Persian Gulf Affairs, National Security Council, in: ebd., 8. suchten die neokonservativen Akteure in der Bush-Regierung zu erschweren, wenn nicht zu unterminieren. John Bolton, einer der unnachgiebigsten US-Repräsentanten, rühmte sich Ende 2007, er habe Außenminister Colin Powell daran gehindert, dass er zusammen mit den Europäern den Iranern Anreize anbot, anstatt auf Sanktionen zu bestehen. ${ }^{41}$

Anfang März 2006 präsentierten die konservativen Realisten ein Iran-Konzept, mit dem sie sich vom neokonservativen Ansatz abgrenzten. Nicholas Burns, Under Secretary im Außenministerium, nannte jetzt die internationale Isolierung der Islamischen Republik als Hauptziel amerikanischer Initiativen in der Nahost-Region. Damit rückten wirtschafts- und finanzpolitische Zwangsmaßnahmen - ein klassisches realpolitisches Instrument - in den Mittelpunkt der konkreten US-Politik. Washingtons Rolle bestand im UN-Rahmen ab Februar 2006 darin, unter den fünf Ständigen Mitgliedern plus Deutschland auf den Beschluss von internationalen Sanktionen zu drängen. ${ }^{42}$ Im Weltsicherheitsrat konnte die Bush-Administration bislang drei Erfolge verbuchen: Im Dezember 2006 verabschiedete das UN-Gremium nach zähen Verhandlungen Resolution 1737. Sie beschränkt den Export bestimmter Materialien und Technologien auf dem Atom- und Raketensektor in den Iran, friert Auslandsguthaben einiger auf diesen Gebieten tätiger Iraner ein und verfügt vereinzelte Reiserestriktionen. Die im März 2007 und 2008 verabschiedeten Entschließungen 1747 bzw. 1803 verschärften im Wesentlichen diese Maßnahmen. ${ }^{43}$ Dabei erwiesen sich Russland und China stets als Bremser.

Da die Regierung Bush die Ergebnisse, die im UN-Rahmen zu erreichen waren, skeptisch beurteilte, setzte sie bereits im Sommer 2006 eine unilateral initiierte Sanktionspolitik in Gang. Führende Repräsentanten des Finanzministeriums versuchten bei ihren Partnern in Banken und befreundeten Regierungen darauf hinzuwirken, ihre Handels-, Investitions- und Finanzierungstätigkeiten mit dem Iran möglichst ganz einzustellen. ${ }^{44}$ Die Islamische Republik sollte so finanz- und wirtschaftspolitisch gelähmt werden. Den betroffenen Banken und Firmen zufolge drohten die amerikanischen Vertreter verdeckt oder unverhohlen mit wirtschafts- und finanzpolitischen Nachteilen bei US-Geschäften. ${ }^{45}$ Im Herbst 2007 zog Washington die Sanktionsschraube weiter an. Die Bush-Administration erklärte die iranischen Revolutionsgarden (Pasdaran), eine ca. 125.000 Soldaten umfassende Eliteformation, und mehrere Banken zu Organisationen, die zur Proliferation von Massenvernichtungsmitteln beitragen. Mit Blick auf die bisherige Wirkung aller Maßnahmen lässt sich jedoch festhalten: Selbst führende

41 The Washington Post, 22.10.2007.

42 Zum Folgenden siehe auch: Bernd W. Kubbig (in Zusammenarbeit mit SvenEric Fikenscher), Internationale Sanktionen gegen den Iran: Erfolgsbedingungen und Effektivität (HSFK-Report 4/2007), Frankfurt/M. 2007.

43 International Herald Tribune, 4.3.2008.

44 So Stuart Levey, Undersecretary of Treasury for Terrorism and Financial Intelligence, in: 110/1 U.S. Congress, Senate, Committee on Banking, Housing, and Urban Affairs: Minimizing Potential Threats From Iran: Assessing the Effectiveness of Current U.S. Sanctions on Iran, Hearing, 21.3.2007 (unautorisiertes Transkript), 40.

45 Financial Times Deutschland, 11.1.2007. 
Befürworter der De-investitionskampagne bewerten den Erfolg der Sanktionspolitik zurückhaltend. ${ }^{46}$

Ausgerechnet das in der "Grand Strategy“ nicht enthaltene Element der Allianzpolitik zur Eindämmung Teherans nahm ab Sommer 2007 einen prominenten Platz auf der außenpolitischen Agenda ein. Ein erstes Indiz für dieses Instrument gab es, als die US-Administration Ende Juli/Anfang August ein umfangreiches Paket von Waffenlieferungen an die unterschiedlichsten regionalen Akteure in zweistelliger Milliardenhöhe ankündigte. Rüstungsexporte an die Verbündeten sind eine traditionelle Komponente amerikanischer Eindämmungspolitik. Die Mitglieder des Golf-Kooperationsrates sollen über einen Zeitraum von zehn Jahren Waffenlieferungen im Wert von rund 20 Mrd. US-Dollar erhalten; weitere 1,3 Mrd. sind für Ägypten vorgesehen, während die jährliche Militärhilfe an Israel von 2,4 auf drei Mrd. US-Dollar erhöht werden soll. ${ }^{47}$ Die USA führen, die arabischen Staaten folgen in der Anti-Iran-Allianz auf Basis „gemeinsamer Interessen“48 - hierin zeigt sich in nuce das realpolitische Modell. Die von Außenministerin Rice auf den Weg gebrachte Nahost-Konferenz in Annapolis vom 27. November 2007 stellte die diplomatische Komponente der „containment“-Politik dar. Für das Gipfeltreffen war es dem State Department mit großem Aufwand gelungen, alle wichtigen regionalen Akteure zu gewinnen. Selbst Damaskus, Teherans wichtigster Verbündeter in der Region, saß mit am Verhandlungstisch und nahm deutliche Dissonanzen mit dem Iran in Kauf. Die Islamische Republik war demonstrativ isoliert.

Präsident Bush setzte den neu eingeschlagenen Kurs im Zuge seiner ersten Reise in den Mittleren Osten/die Golfregion Anfang 2008 fort. Beide Elemente, ausgeprägter Dialog mit den Verbündeten vor Ort und die konkreten Rüstungsofferten, standen im Fokus von Bushs Gesprächen mit maßgeblichen Akteuren, deren Repräsentanten zur Annapolis-Konferenz gereist waren. In seiner programmatischen Rede in Abu Dhabi vom 13. Januar 2008 war nicht mehr die Demokratisierung der Region das Hauptthema. Stattdessen proklamierte Bush das Ziel, die „gemäßigten Kräfte“ in der Region zu stärken und den negativen Einflüssen des Irans, Syriens, der al-Quaida sowie der Hezbollah entgegenzuwirken. Bush sprach nicht von einem unmittelbaren „regime change“ im Iran, sondern thematisierte die Demokratisierung im Sinne einer Langzeitstrategie mit den Iranern als Akteuren, die den Wandel selbst voranbringen sollen. ${ }^{49}$ Auf der konzeptionellen Ebene zeigen Annapolis und Bushs Reisediplomatie den Ausbruch der Administration aus

46 Siehe die Aussagen von Levey vor dem Kongress (110/1 U.S. Congress, Senate, Committee on Banking, Housing, and Urban Affairs: Minimizing Potential Threats From Iran: Assessing the Effectiveness of Current U.S. Sanctions on Iran, Hearing, 21.3.2007 [unautorisiertes Transkript], 40) Ähnlich fällt auch die neueste offizielle Einschätzung vom Frühjahr 2008 aus (siehe das Statement von Daniel Glaser, Deputy Assistant Secretary of Treasury for Terrorist Financing and Financial Crimes, in: 110/2 U.S. Congress, House of Representatives, Committee on Foreign Affairs, Subcommittee on the Middle East and South Asia/Subcommittee on Terrorism, Nonproliferation and Trade: Preventing Nuclear Iran, Joint Hearing, 17.4.2008 [unautorisiertes Transkript], 18)..

47 So die Ausführungen von Unterstaatssekretär Burns bei einer Pressekonferenz am 6.8.2007 (U.S. Department of State, www.state.gov/p/us/rm/2007/90347. htm [letzter Zugriff: 4.9.2007]) und die Angaben in The Washington Post vom 28.7.2007.

48 So US-Außenministerin Rice (BBC News, www.news.bbc.co.uk/1/hi/world/ middle_east/6923430.stm [letzter Zugriff: 3.9.2007]).

49 The White House, Office of the Press Secretary, President Bush Discusses Importance of Freedom in the Middle East, www.whitehouse.gov/news/releases/2008/01/print/2008.113-1.html (letzter Zugriff: 21.1.2008). der neokonservativ ausgerichteten „Grand Strategy“. Auch auf der Ebene praktischer Politik demonstrieren beide Ereignisse, dass sich die von Condoleezza Rice angeführten Vertreter realpolitischer Konzepte im Aufwind befinden.

\section{Die Iran-Politik der Administrationen Clinton und Bush: Partielle Rückkehr Bushs zu den traditionellen Elementen}

Der Fokus auf den Aspekten Kontinuität und Neuerungen ermöglicht es, sowohl die unterschiedlichen Ziele als auch die gesamte Bandbreite außenpolitischer Instrumente der beiden US-Administrationen in den Blick zu nehmen. Die Clinton-Administration war im Rahmen ihres realpolitisch ausgerichteten Gesamtkonzepts, das sich in der „Nationalen Sicherheitsstrategie“ von 1995 niedergeschlagen hatte, mit dem Ziel angetreten, das Verhalten des Regimes in Teheran entsprechend der US-Interessen zu beeinflussen. Das hauptsächliche Instrument war mit dem „dual containment" eine besondere Form der traditionellen Eindämmung, die in der politischen Praxis mit der Bereitschaft zum Dialog und mit Angeboten der Annäherung an Teheran verbunden war.

Auf der Ebene der „Grand Strategy“ stellen die „Nationalen Sicherheitsstrategien“ von 2002 und 2006 einen Bruch mit der NSS der Clinton-Regierung dar. Die ausgerufene Abkehr der amerikanischen Iran-Politik von realpolitischen Prinzipien zu Gunsten der „Freiheits-Agenda“ bestimmte unter dem Einfluss der Neokonservativen die Formulierung der Gesamtstrategie. Im Sinne der eingangs differenziert gestellten Forschungsfrage setzte sich dieses Gesamtkonzept - und mit ihm die FreiheitsVision - bis gegen Ende der Bush-Ära jedoch nicht in der praktischen Politik durch. Denn den „Neocons“ gelang es nicht, die US-Politik an ihrem Ziel, in Teheran notfalls mittels Gewalt im Sinne der Bush-Doktrin einen Regimewechsel herbeizuführen, auszurichten. Selbst die beschlossenen Maßnahmen zur Demokratieförderung standen letztlich im Zeichen realpolitischer Überlegungen. Kurzum, auf allen drei Ebenen - Gesamtstrategie, außenpolitische Ziele und Einsatz der Mittel - wurde die Iran-Politik der Neokonservativen zur Episode.

Anfang 2006 präsentierten die konservativen Realisten ihr eigenes Konzept. Damit rückte der weltordnungspolitische Grundsatz der Stabilität in den Mittelpunkt; das Ziel war jetzt die internationale Isolierung der Islamischen Republik sowie, gekoppelt mit der angestrebten Schwächung des Regimes, die langfristig angelegte Demokratisierung des Irans; bei den Instrumenten verlagerte sich die Präferenz nun auf Sanktionen und Allianzpolitik zur Eindämmung Teherans. Mit diesen Elementen klassischer Realpolitik traten Rice und Gates aus dem Schatten der „Grand Strategy" heraus. Gleichzeitig knüpften sie an die realpolitische Tradition der Clinton-Ära an, dies jedoch nur partiell. Denn ihrer Eindämmungspolitik fehlte das Element der selektiven Einbindung des Irans. Damit fiel die Bush-Administration hinter die Ende der 1990er Jahre modifizierte Agenda der Regierung Clinton und ihr bedingungsloses Gesprächsangebot zurück. Anders als ihre Vorgänger lehnten die Vertreter der Bush-Regierung Kooperationsangebote aus Teheran ab. Bis gegen Ende der Ära Bush weigerten sie sich, selbst mit einem Moderaten 
wie den früheren Präsidenten Chatami zusammenzutreffen. Eine weitere Diskontinuität ist in der Demokratisierungsfrage zu konstatieren. In Clintons Konzept war primär die Stabilisierung der neu etablierten freiheitlichen Systeme in Osteuropa und Russland vorgesehen. Die Islamische Republik fand in der Demokratisierungspolitik keine Berücksichtigung.

Dass sich in der Ära Bush die konservativen Realisten mit ihrem Konzept bislang durchsetzten, ist vor allem auf die nach wie vor katastrophale Lage im Irak zurückzuführen, die amerikanische Streitkräfte in großem Umfang bindet. Die Entwicklung im Zweistromland beeinflusste die Kräfteverhältnisse und Positionen in den USA und in seinem Entscheidungssystem beträchtlich. Hierzu zählen die Ausdünnung der neokonservativen Gruppierung sowie die Abkehr vom Ziel des Regimewechsels und, damit verbunden, die vorherrschende Unterstützung von Sanktionen im Kongress. Ein besonders wichtiger Faktor war die Teilentwarnung durch alle 16 Geheimdienste, dass von Teheran keine akute Bedrohung ausgehe.
Gleichzeitig ist der Iran aufgrund der Lage im Irak selbstbewusster geworden. Das Atomproblem ist lediglich vertagt. Denn auch das Konzept der konservativen Realisten war bisher nicht erfolgreich. Teheran hat die Hauptforderung, bei der Urananreicherung einzulenken, keinesfalls erfüllt. Verlässliche Schlussfolgerungen lassen sich aus dem herausgearbeiteten Trend und seinen Determinanten nicht ziehen. Zum einen nehmen die beiden Präsidentschaftskandidaten - John McCain und Barack Obama - unterschiedliche Positionen in der Iran-Frage ein. Zum anderen wird jeder Kandidat seinen IranKurs als Präsident nicht in einem inneramerikanischen sowie regionalen und internationalen Vakuum festlegen. Es lässt sich nicht vorhersagen, ob die herausgearbeiteten maßgeblichen Determinanten weiterhin politikmächtig bleiben und die Präferenzen im Handlungsrepertoire des nächsten Präsidenten und seiner Administration mitbestimmen. Da die in der Ära Bush ungelösten Probleme mit dem Iran nur vertagt sind, bleiben die Handlungsoptionen für die nächste Administration zwischen den Eckpunkten Einäscherung iranischer Anlagen und Einbindung des Regimes in Teheran bestehen.

\title{
Sicherheit auf See
}

\section{- Risiken weltweit und in deutschen Hoheitsgewässern}

\author{
Uwe Jenisch*
}

\begin{abstract}
Safety and security at sea are a key issue for all maritime states. Traditionally, maritime law has included a well developed body of safety rules, including rules for the management of environmental risks. However, security risks have increased recently due to piracy, terrorism and organised crime. This article analyses the full spectrum of modern maritime security risks and the relevant legal instruments that are already in force or are in the process of being negotiated. Apart from the legal aspects, control and enforcement at sea, i.e. an efficient coast guard with the competence and the power to intervene, are crucial when terrorists or pirates attack. The role of navies versus "police-type" organisations is also at stake. Detailed emergency plans exist to deal with major accidents and environmental disasters in German waters, but a centralised coast guard service with preventive and executive powers is still under discussion, while the German constitution prevents the Navy from intervention.
\end{abstract}

Keywords: Maritime Sicherheit, Deutsche Küstenwache, Deutsche Marine

\section{Vorbemerkung}

$\mathrm{D}$ as Thema maritime Sicherheit behält zentrale Bedeutung. Spektakuläre Unfälle wie der Untergang der Fähren 2006 im Roten Meer ${ }^{1}$ und in Indonesien mit zusammen rund 1.400 Opfern, der Brand hunderter von Containern auf der „Hyundai Fortune“ im März 2006 vor Jemen, der spektakuläre Untergang des Kreuzfahrers „Sea Diamond“ im Hafen von Santorin am 6. April 2007 oder die Strandung der „MSC Napoli“ im Januar 2007 an der britischen Kanalküste mit nachfolgender mühseliger Bergung von 4.700 Containern sind überall möglich. Das gilt auch vor den deutschen Küsten, wo sich die Schifffahrtswege mit jährlich rund 200.000 Schiffs-

\footnotetext{
* Uwe Jenisch, Prof. Dr. jur., Walther Schücking Institut für Internationales Recht, Universität Kiel. Dieser Aufsatz wurde referiert (peer-review).

1 Untergang der ägyptischen RoRo Fähre „Al Salam Boccaccio 98“ am 03.02.2006 mit ca. 1.000 Toten, Flagge: Panama. Untergang „Senopati Nusantara“, Dez. 2006 in Indonesien mit 400 Opfern
}

bewegungen für Nordeuropa und für die gesamte Ostsee konzentrieren.

Für die betriebliche Sicherheit von Schiffen („safety“) besteht ein immer engeres Netz von Regelungen durch die Weltschifffahrtsorganisation IMO und die EU. Gleichzeitig treten äußere Bedrohungen wie Piraterie, Kriminalität und Terror in den Vordergrund - ein Bereich, der international mit „security“ umschrieben ist. Zusätzlich sind mit Blick auf den Klimawandel vermehrte Naturkatastrophen an den Küsten nicht auszuschließen. Anders als im Englischen gibt es in der deutschen Sprache nur einen Sicherheitsbegriff. Das unterstreicht, dass Sicherheit unteilbar ist. Nicht zuletzt aus diesem Grund plädiert dieser Beitrag für einen einheitlichen maritimen Sicherheitsdienst. Allen Fachleuten ist klar: Eine absolute Sicherheit gibt es nicht. Auch den Besten kann das schlimmste Unglück treffen.

Trotz gelegentlicher dramatischer Schlagzeilen in den Medien ist der Seetransport insgesamt betrachtet der umweltfreund- 